\title{
A Performance Analysis of Wavelet based LTE-OFDM with Multi- equalizers
}

\author{
Judy Simon*1, N Prabakaran² \\ ${ }^{* 1}$ Research Scholar, Faculty of Electronics, Sathyabama Institute of Science and Technology, Chennai \\ ${ }^{2}$ Associate Professor, Department of ECE, Koneru Lakshmaiah Education Foundation, Vaddeswaram, Andhra \\ Pradesh, India \\ *Correspondence: judyminisha@gmail.com
}

Article History:Received:11 november 2020; Accepted: 27 December 2020; Published online: 05 April 2021

\begin{abstract}
A rapid change in usage of mobile data and mobile based applications such as online gaming, mobile TV etc. motivates the mobile wireless communication system to work on new emerging trends Long Term Evolution (LTE). LTE-4G architecture provides an optimized packet based radio access technology. The basic signal format of LTE is Orthogonal Frequency Division Multiplexing (OFDM) Modulation. This modulation scheme satisfies the spectrum flexibility requirement in LTE. LTE-MIMO-OFDM with IFFT and FFT transform introduced to provide the cost-effective solution for very wide carriers with high PAPR. Inter-symbol Interference (ISI) and inter carrier interference (ICI) are predominant factors which exist in the wireless communication channel. Existing methods of ISI and ICI reduction to improve signal quality have been achieved using conventional OFDM techniques. However, a better modulation scheme is required to achieve high data rate, low latency and flexible bandwidth deployment. This paper has proposed a Wavelet based LTEMIMO-OFDM modulation scheme with multi equalizer that improves the performance of system by reducing PAPR and BER in MCM. Experimental results show the performance of this scheme and the results is compared with existing MMSE and Multi equalizer MMSE and achieves a flexible spectral efficiency with reduction of PAPR and BER.
\end{abstract}

Keywords: Multiple Input Multiple Output - Orthogonal Frequency Division Multiplexing, Discrete Wavelet Transform - Orthogonal Frequency Division Multiplexing, Long Term Evolution, Multi-equalizers, Minimum Mean Squared Error, MLE, Bit Error Rate, Additive White Gaussian Noise.

\section{Introduction}

Wireless communication systems [1-2] have been exploited widely across the world in present days, due to its requirements as part of even a small business. The wireless communication systems WI-FI, WiMAX etc. adopted various IEEE 802.11 wireless standards and LTE/LTE-A cellular communication standards [3-4] to achieve faster transmission with high data rate and flexibility in data accessing. The wireless communication system suffers from the problem of interference of signals whenever signals are transmitted through open communication medium like air. A technology that minimizes the interference of frequency signals among channels, and achieves clean data transmission has been obliged. Orthogonal frequency division multiplexing (OFDM) [5-6] has been introduced to replace the existing conventional modulation scheme. OFDM is a modulation technology which has the tendency of reducing signal corruption and interference of signals, so that it overcomes the performance of the conventional modulation technology.

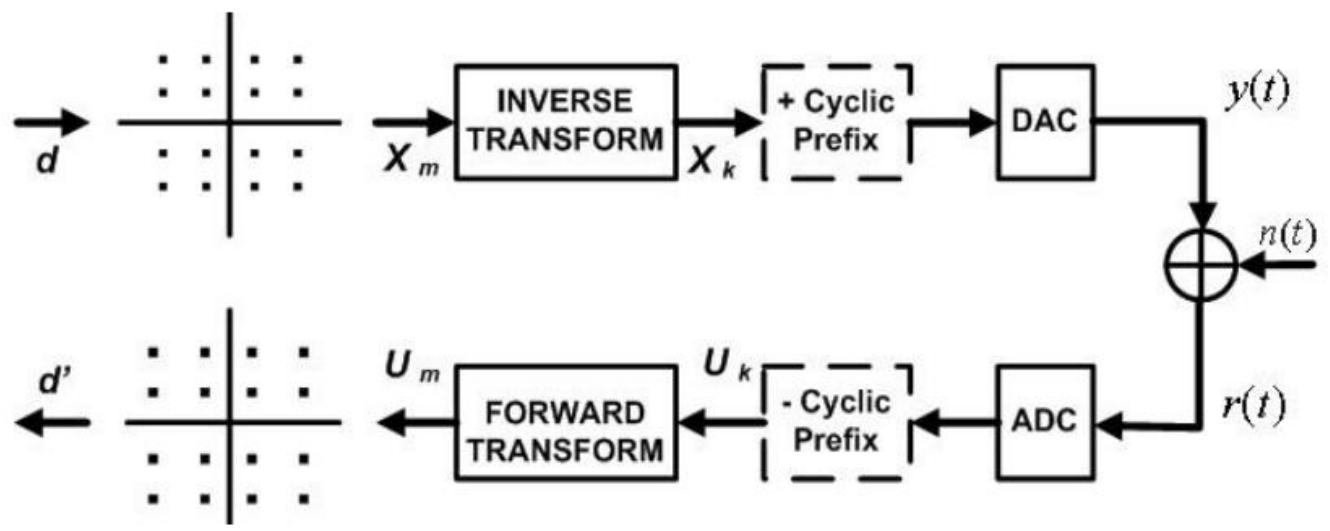

Figure 1. Basic Model of OFDM

OFDM is a multiple carrier frequency modulation scheme, in which a high data rate stream is divided across sub carriers at different frequencies. The subcarriers are orthogonal to each other so that the signals in one carrier do not interfere with other carrier. The general basic system model of OFDM is shown in Figure 1.

*Correspondening Author: JUDY SIMON

108

Research Scholar, Faculty of Electronics, Sathyabama Institute of Science and Technology, Chennai judyminisha@gmail.com 
In conventional OFDM [7], Inverse Fast Fourier Transform (IFFT) introduced for multiplexing the signal together at the transmission end and the multiplexed transmitted signal is enhanced with the cyclic prefix and then the resulting signal is transmitted to the carrier. At the receiving end, the cyclic prefix is removed from the received signal and then the signals are de multiplexed using Fast Fourier Transform [8] (FFT). The adding of cyclic prefix has the disadvantages of affecting the performance of data throughput and power efficiency even though the purpose of adding cyclic prefix is to diminish inter symbol interference (ISI). The characteristics such as multi-carrier modulation and introduction of guard symbol at each symbol make OFDM free from ISI transmission.

The main problems that influence the performance of the conventional modulation scheme are InterSymbol Interference (ISI) and Inter-Carrier Interference (ICI). Deformation in the transmission signal is called Inter Symbol interference. Deformation, one signal is interfering with consequent signal, is happened due to the multipath propagation. Multi propagation occurs due to transmission of signals through many reflected and diffracted surroundings. Some signals follow the direct path and others follow the reflected path to reach the receiver so that the transmitted signal are slightly out of phase and causes different propagation time for the transmitted signal at the receiver side. The vector value of multipath propagated signals is combined at the receiver end and produces a signal with small scale frequency fading. This causes the inter symbol interference. The carrier frequency offset and sampling frequency offset are the main reasons for interference of signals of one subcarrier to another called ICT. Doppler spread and imperfect synchronization offset of signals causes distortion within the same signal. A promising technique is required to handle the situation of time dispersive nature of wireless channels occurs due to inter symbol interference and losing of orthogonal property occurs due to inter carrier interference. The popular technology in $4 \mathrm{G}$ wireless mobile network that can minimize the inter symbol interference and inter carrier interference effect and achieves high data transmission is known as OFDM.

The feasibility of the wireless communication is highly affected by factors of throughput, data rate, Bits Error Rate (BER) and the quality of the signal received at the receiving end. These parameters are mainly estimated by the number of the antennas available at the transmitting and receiving end and, also based on the noise introduced in the system due to the external disturbance. MIMO based OFDM gets attraction in wireless communication system now a day. MIMO uses spatial domain multiplexing that combine with orthogonal frequency division multiplexing in order to provide best signal transmitter and receiver diversity gain.

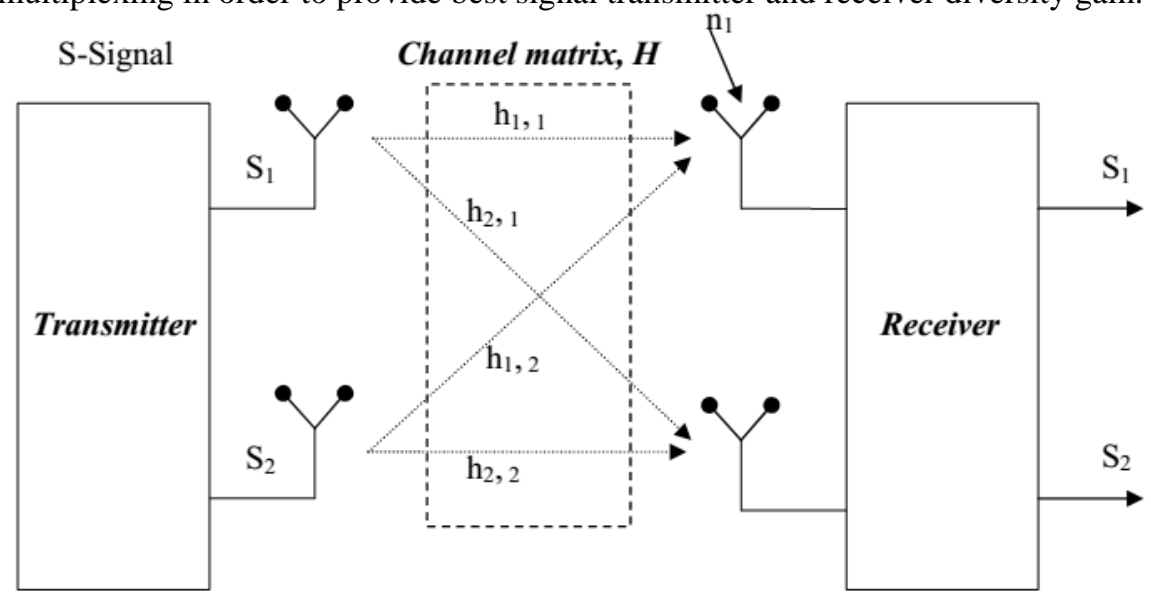

Figure 2. Structure of MIMO System

The optimal value of BER is maintained by the multiple antennas at both receiving and transmitting ends. The MIMO-OFDM produces high bandwidth efficiency so that MIMO-OFDM has been used as a potential candidate for broadband wireless access.

OFDM with DFT has limitations in achieving high Peak to average power ratio of the transmitted signal due to amplitude variation. The amplifier will occasionally reach the amplifier saturation region by indicating large peaks results in signal distortion. The amplifier that is linear and tolerates highest amplitude variations is required but it has the drawback in terms of efficiency. Even though OFDM eliminates the time delay [8] by introducing the guard interval, a different form of time delay experienced by OFDM due to the difference between the first and last delayed path which is greater than the guard interval introduced. This excess delay difference causes both ISI between two adjacent signals and ICI within the same signal in OFDM system. To overcome these issues, the wavelet based transform [9] with DWT is introduced in OFDM in place of FFT. DWT-OFDM with equalization technique is discussed to reduce the Peak to average ratio and reduces the interference of the channel [10] especially in the case where the excess delay is more than one half of the signal duration. The paper is organized as follows: Section II produces the survey of the related works. Section III 
discusses the proposed methodology and Section IV makes an analysis of experimental results. Section V concludes.

\section{Related Works}

In multipath channel, conventional OFDM employs IFFT based operations for mapping the signal and Cyclic Prefix (CP) augmented with the transmitted signal to reduce the inter symbol interference and then the transmitted signal is generated for transmission. The CP added at the transmission end leads to the problem of power loss by degrading the spectral efficiency of the channel. Conventional OFDM has drawback of producing high Peak-to-Average Power ratio (PAPR). The PAPR can be reduced by introducing IDWT, DWT instead of IFFT, FFT. The wavelet-OFDM [11-12] based on the sine, cosine functions and number of wavelets is proposed. The wavelet transformation with various wavelets and the corresponding signal changes for each wavelet is studied. The performance of the wavelet-OFDM and its various characteristics is analyzed. The wavelet-OFDM has same bit error rate (BER), less Out-Of-Band (OOB) and less PAPR than the conventional OFDM. The discrete based DWT-OFDM, does not uses CP [13-14] and thereby it improves the spectral efficiency. The channel is divided into sub channel and its attributes are decided to improve the sun channel spectral efficiency [15-16].

The performance of DWT-OFDM is analyzed under AWGN environment and Saleh-Valenzuels [17] channel environment is proposed. The PAPR and BER performance is compared with existing DCT-OFDM and DFT-OFDM. The orthogonal property of the channel is maintained by introducing cyclic prefix to the OFDM frame. In DFT-OFDM, the pilot subcarrier at regular intervals is inserted into the received distorted signals so that the received signals are equalized. The equalization technique at the received signal improves synchronization between the signals of transmitter and receiver. The Zero padded guard intervals [18], that is, inserting zeros at the transmitted signal, is introduced to reduce ISI in both DCT, DFT, thereby resulting in increase of the channel efficiency. Discrete Cosine transforms and Discrete Fourier transforms have been found to give ideal and promising solutions into reducing the BER with the former having a marginally superior performance over the latter. Amongst, DFT-OFDM based methods, zero padded techniques exhibit a superior performance over cyclic prefix (CP) based methods. In DWT-OFDM system, the various type of wavelet like Haar, Daubechies, Symlet, biorthogonal are studied and their performances are analyzed. In AWGN channel environment, Haar, Daubechies produces lower bit error rate compared to DFT-OFDM. In Saleh-Valenzuels environment, DFT-OFDM produces better performance.

The time domain based zero forcing equalization for comparing the performance of wave let based MCM and FFT-OFDM [19] is proposed. The wavelet implemented under BPSK signal mapping and AWGM based multipath channel environment is analyzed. The wavelet uses orthogonal filters called Quadrature Mirror Filter (QMF) bank. The QMF consists of two filters called low pass and high pass that supports to decompose the signals into low band and high band signals of equal length. The BER performance is improved by having side loops that restricts out of band emission. The multi resolution analysis is made for localizing the time, frequency and resolution using the transformation technique called shifting and scaling. A simple zero forcing equalization is applied. The channel state is known to the receiver, so that the fair equalization is obtained by removing the channel error introduced due to the noise.

Minimum mean square error based equalizer algorithm is proposed to wavelet-OFDM to study its performance based on Meyer wavelet under AWGN channel environment. Frequency domain equalization is applied to reduce BER. Minimum mean square error equalizer also applied to reduce PAPR as well as BER at the cost of computational complexity by up to 4.5-6.5 dB of SNR. In order to reduce the computational complexity experienced by MMSE due to the Doppler frequency of sub carrier, a multi-segmental equalizer with Piecewise Linear Model (PLM) [20] is proposed. The complexity of the equalizer is minimized by using small sized segments. The performance improved by increasing the number of segments. Multi-segmental equalizer replaces MMSE by updating the channel matrix.

A discrete DFT-OFDM system with 4-Quadrature Amplitude Modulation (4QAM), cyclic prefix and equalization in AWGN environment is used to study the BER performance. The different equalizer, Zero forcing (ZF) equalizer and Minimum Mean Squared Error (MMSE) equalizer [21], can be implemented at the receiver side to reduce the interference is proposed. Zero Forcing equalization is the technique of applying the frequency response in inverse. Let $\mathrm{R}(\mathrm{f})$ be the frequency impulses response of the channel, then zero forcing equalizer $\mathrm{C}(\mathrm{f})$ is calculated using the formula

$$
C(f)=1 / R(f)
$$

If the frequency of the signal receive is weak, then the zero forcing equalizer will increase in magnitude. At the same time, any noise in the channel is also increased in greater magnitude, thereby it affects overall SNR. In order to reduce the time dispersive nature of the signal and the noise effects added to the signal, minimum mean square equalizer is introduced to optimize the signal value. The ISI interference and noise is not removed fully, but the power can be reduced. The modulation system is simulated with three channels and with introducing, without introducing two equalizers. After using each equalizer, the BER performance and symbol constellation 
is measured. The BER performance for Single Carrier-FDMA [22] against four channels AWGN, Rayleigh, Pedestrian A and Vehicular A channels analyzed. Zero Forcing and MMSE equalizer is implemented with SCFDMA, IFDMA and their performance are studied.

At the transmission end, different Crisp Factor Reduction techniques called clipping and filtering, windowing, and cancellation is used to reduce PAPR and BER. At the receiver end, the equalizer is introduced at the distorted signal, so that the PAPR and BER can be reduced. The equalizer can be categorized into Linear and Nonlinear. MCM with linear equalizer is well studied and analyzed. If the given channel is noisier and not able to equalize the channel distortion due to the severe noise, then the nonlinear equalizer is used. This paper proposed a Wavelet based OFDM with Zero Forcing and Maximum Likelihood Estimation equalizer method to reduce the BER in an attempt to improve the overall communication system performance over the Gaussian Channel characterized with white noise.

\section{Proposed Work}

The proposed architecture introduces the Wavelet based MIMO-OFDM improves the spectral efficiency of sub channel containment by minimizing the interference between the symbols. A novel method of improving the communication system performance by reducing BER is utilized in this research work by exploiting OFDM using wavelet transforms. The basic system model of DWT-OFDM with two equalizers is shown in Figure 3. The DWT-OFDM consists of two basic components, DWT-OFDM transmitter and DWT-OFDM receiver.

Input

signal (d)
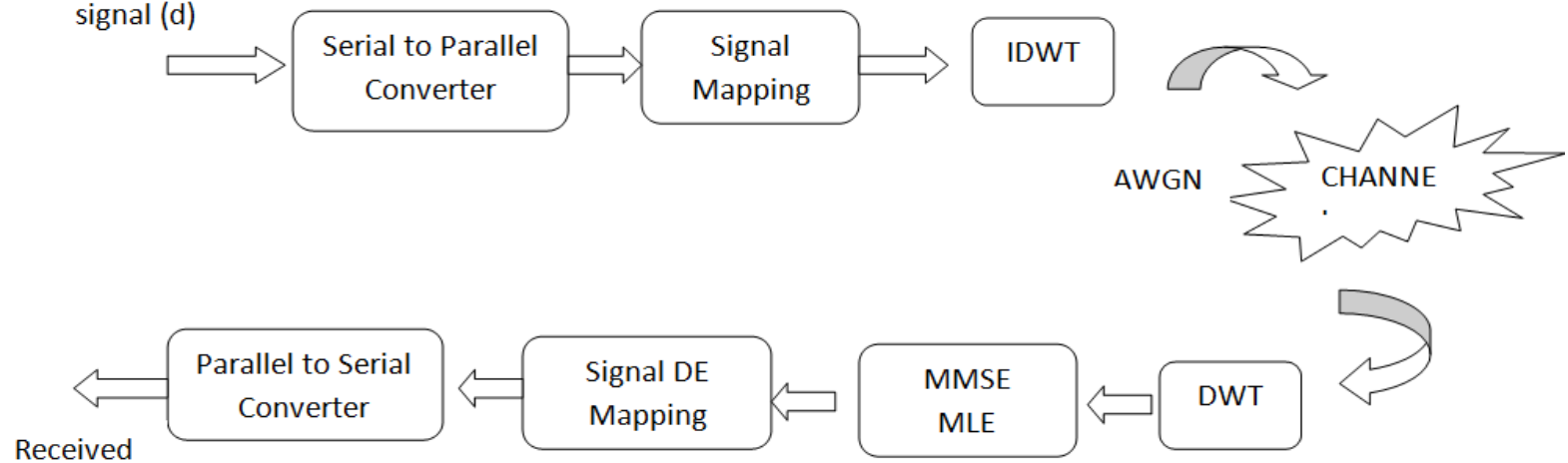

Signal

a) DWT transmitter

Figure 3. Basic Model of Wavelet OFDM transform

The DWT transmitter model is shown in Figure 4. It receives the serial bits of data $\mathrm{d}$ as input. The serial to parallel converts the given input $d$ into $\mathrm{N}$ parallel data stream $\mathrm{X}_{\mathrm{N}}$. The 1 mapping of the serial bits $\mathrm{d}$ to one of the $\mathrm{X}_{\mathrm{m}(\mathrm{i})}, 0 \leq \mathrm{i} \leq \mathrm{N}-1$ parallel data stream is obtained by QAM modulator. Ortho-normal wavelet is the basis for discrete wavelet transform. Each $\mathrm{X}_{\mathrm{m}(\mathrm{i})}$ is converted into serial representation using parallel to serial converter and produces a vector data $\mathrm{X}_{\mathrm{x}}$. The vector data is then transposed which is actually a negative response of the input but it can produce as a parallel matrix $\mathrm{X}_{\mathrm{A}}$.

$\mathrm{X}_{\mathrm{m}(0)}$
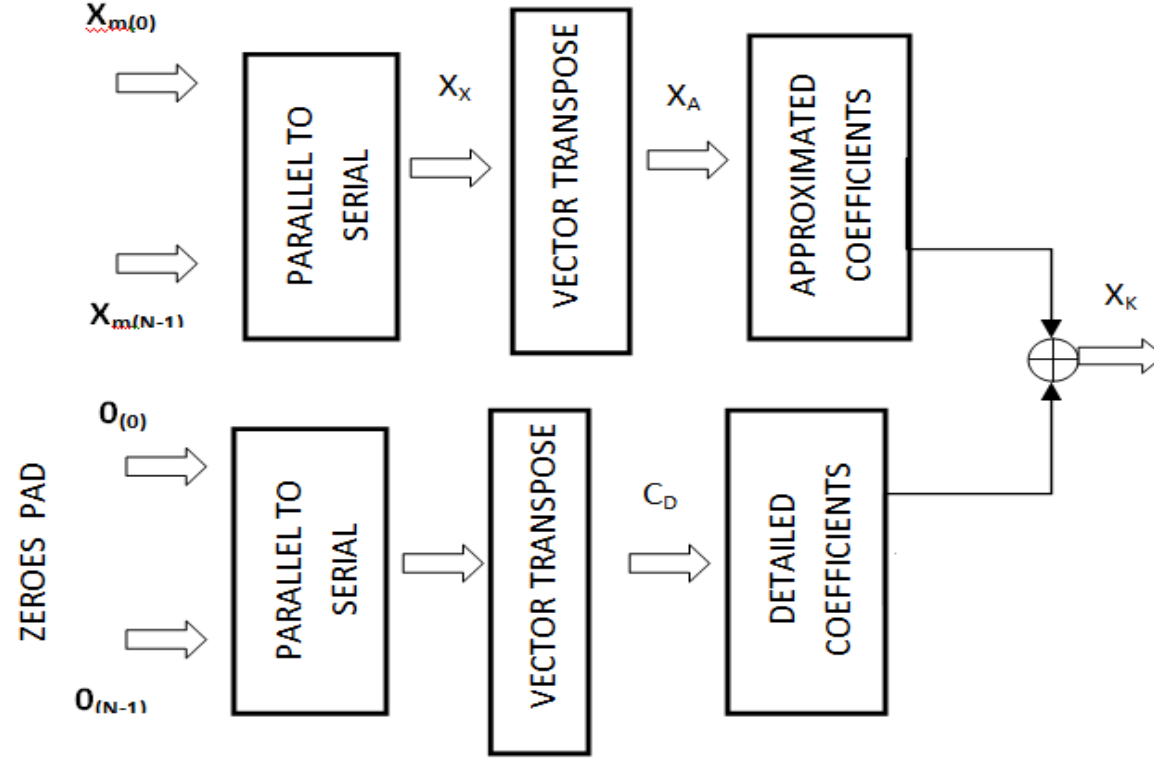
Figure 4. DWT Transmitter

The signals are approximated to produce approximate coefficient by convolution of the signals using low pass filter that produces low frequency signal. At the same time, Zeroes padded signals convolved with high pass filter produces the detailed coefficient. The approximate and detailed coefficient combined to satisfy Orthonormal base as wavelet transform. The combined signals are transmitted through the carrier of the channel.

b) DWT receiver

The signal received from the channel is decomposed into approximate and detailed coefficients using two filters banks called low pass filter and high pass high pass filter. The output of the approximated coefficients $\mathrm{X}_{\mathrm{A}}$ will be processed by applying vector transpose and parallel to serial converter to get $\mathrm{V}_{\mathrm{m}(\mathrm{i})}$. The signals received from detailed coefficient $C_{d}$ is discarded when compared with $\mathrm{X}_{\mathrm{A}}$, since it does not have any useful information

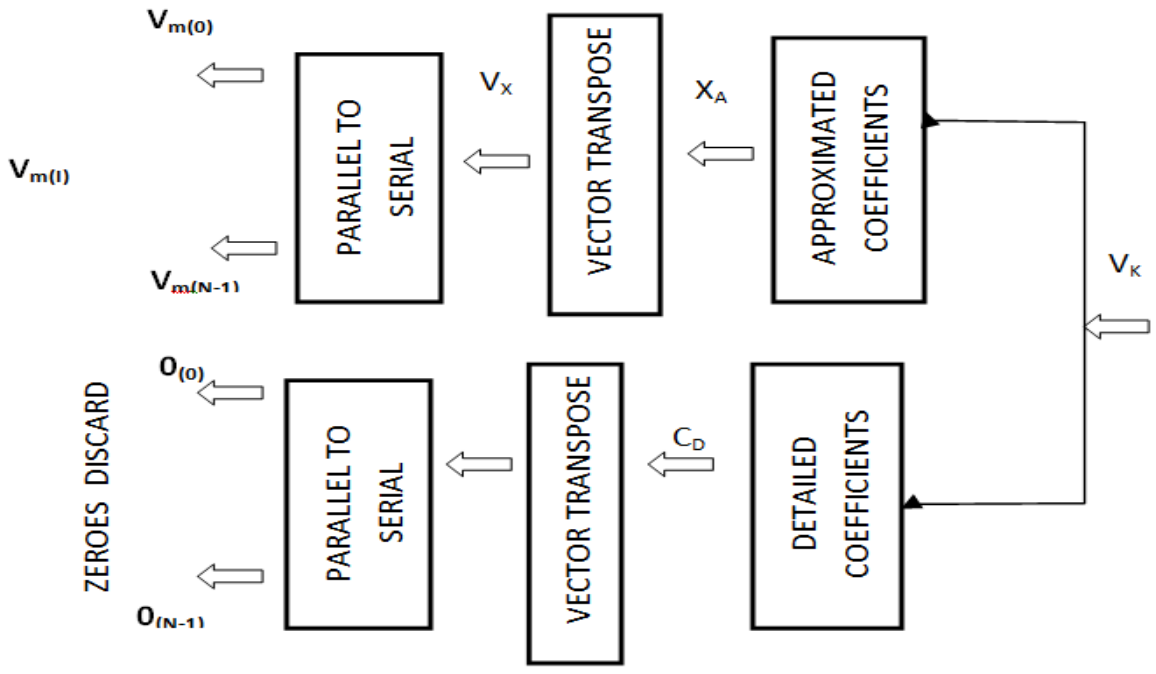

Figure 5. DWT Receiver

c) Wavelet Carrier

In DWT-OFDM based Haar wavelet, the wavelet carrier is denoted by a function $\psi$ at different level of scales $l$ with time axis $\mathrm{x}$ is defined in equation 2

$$
\varphi_{\mathrm{l}, \mathrm{x}}(\mathrm{t})=2^{-1 / 2} \varphi\left(2^{-1} \mathrm{t}-\mathrm{x}\right)
$$

The Orthogonality feature of the carrier is expressed by generating wavelet family at time location $t$ is given in equation 3

$$
\left(\varphi_{\mathrm{l}, \mathrm{x}}(\mathrm{t}) \varphi_{\mathrm{m}, \mathrm{n}}(\mathrm{t})\right)=\left\{\begin{array}{c}
1 \text { if } \mathrm{l}=\mathrm{m} \text { and } \mathrm{x}=\mathrm{n} \\
0 \text { otherwise }
\end{array}\right.
$$

DWT-OFDM is expressed as the sum of wavelet with its weight and carrier scale coefficient is denoted as in equation 4

$$
\mathrm{s}(\mathrm{t})=\sum_{\mathrm{l} \leq \mathrm{l}} \sum_{\mathrm{x}} \mathrm{w}_{\mathrm{l}, \mathrm{x}}(\mathrm{t}) \varphi_{\mathrm{l}, \mathrm{x},}(\mathrm{t})+\sum_{\mathrm{x}} \mathrm{a}_{\mathrm{l}, \mathrm{x}}(\mathrm{t}) \varphi_{\mathrm{l}, \mathrm{x}}(\mathrm{t})
$$

where $\mathrm{w}_{\mathrm{ll}, \mathrm{x}}$ denoted the sequence of the wavelet and $\mathrm{a}_{\mathrm{l}, \mathrm{x}}$ denotes the approximation coefficient

d) Estimation of channel coefficient

Channel coefficient can be estimated by the concept of de-convolution over the received signals. The received distorted signal is de-convolved with the already existing copy at the receiver. Multi equalizer, Minimum Mean Square Error (MMSE) and Maximum Likelihood Estimation (MLE), are used to estimate channel coefficients and equalizes the distorted signal with transmitted signal. The objective of MMSE estimator is to reduce the ISI. MMSE estimator objective is to find the best filter coefficients that produces smallest error signal in the mean square. MMSE is a quadratic function that has only one extreme that minimizes the mean square error. The algorithm works based on the error at the current channel time. The objective is to find best optimal filter design. At the initial step the algorithm stats with smallest filter weights values. At each step, the gradient of the mean square error find out and weights updated. The weight updates in the direction opposite to the gradient slope is given in equation (5)

$$
\mathrm{W}_{\mathrm{n}+1}=\mathrm{W}_{\mathrm{n}}-\partial \Delta \epsilon[\mathrm{n}]
$$

Where $\in$ is the mean square error and $\partial$ is the constant step size. The mean-squared error function is defined as

$$
\in[\mathrm{n}]=\mathrm{E}\left(\mathrm{er}^{2}(\mathrm{k})\right)
$$

where $\operatorname{er}(\mathrm{k})$ is the error signal calculated as

$$
\mathrm{er}(\mathrm{k})=\mathrm{d}(\mathrm{k})-\mathrm{r}(\mathrm{k})
$$


Where $\mathrm{d}(\mathrm{k})$ is the desired signal and $\mathrm{r}(\mathrm{k})$ is the output of the equalizer which calculated by taking the coefficient vector from the delay line as input.

MLE equalizer is used to reduce the nonlinear impairments introduced by the channel. It works based on the Expectation and Maximization algorithm. It is two step iterative algorithms that repeat expectation and maximization step until the estimate converge. In EM algorithm, the initial estimate of the channel is denoted by $\hat{h}(0)$. The ML estimate of $h$ is

$$
\hat{\mathrm{h}}=\operatorname{argmax}_{\mathrm{h}}(\log (\mathrm{P}(\mathrm{r} \mid \mathrm{h})
$$

Where $\log (\mathrm{P}(\mathrm{r} / \mathrm{h})$ is known as probability density function of the received sequence $\mathrm{r}$, for given channel impulse responses is calculated as

\section{Results and Discussion}

$$
\log (\mathrm{P}(\mathrm{r} \mid \mathrm{h})=\log (\mathrm{P}(\mathrm{r} \mid \mathrm{a}, \mathrm{h}) \cdot \mathrm{p}(\mathrm{a}))
$$

The simulation is carried out in MATLAB under the DWT-OFDM based on Haar wavelet with 16 QAM modulation scheme under the AWGN channel environment. The performance of DWT-OFDM is analyzed with different equalizer in the presence of AWGN environment. The DWT-OFDM with MMSE and MLE equalizer is compared against DWT-OFDM with MMSE and DWT-OFDM with multi equalizer Zero Forcing (ZF), MMSE. The performance factors PAPR, BER, upload throughput, download throughput and spectral efficiency against SNR are analyzed.

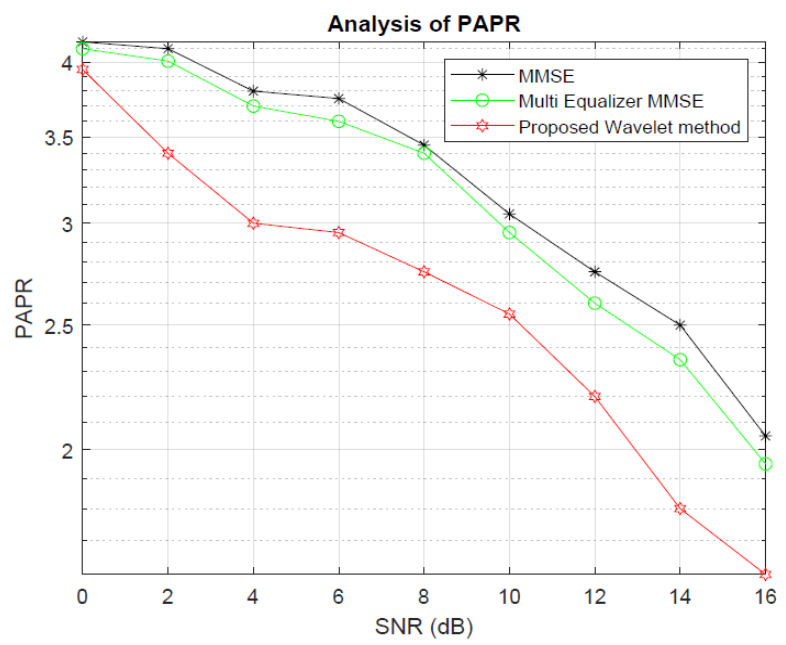

Figure 6. PAPR vs SNR

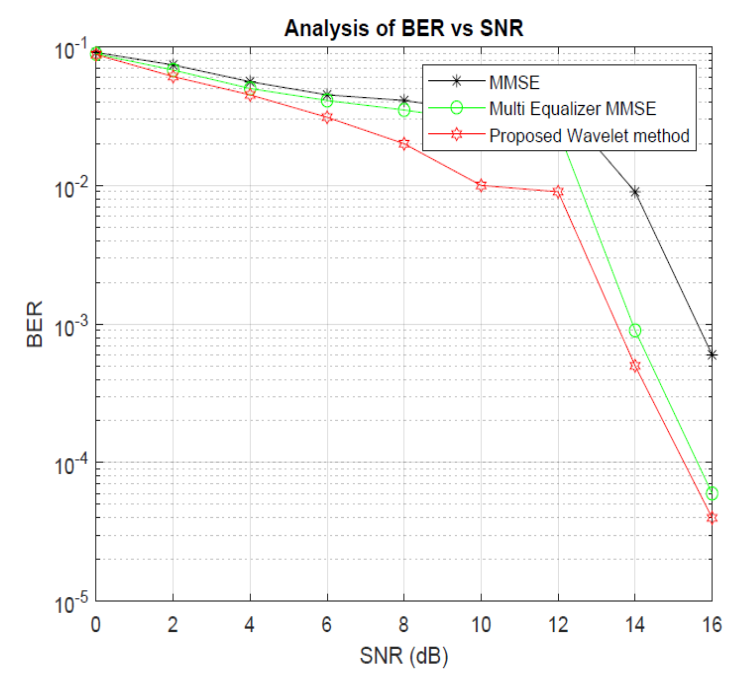

Figure 7. BER vs SNR

The number of scales $\mathrm{L}$ of the wavelet transform decides the PAPR performance against SNR. If the scale is high, then complementary cumulative distribution function shifted right in time that causes degradation in PAPR. The multicarrier system can be viewed as a single carrier system since they have the same scale, same bandwidth and only differ in the shifting in time. The Figure 6 shows that the PAPR performance of the proposed wavelet has less rate effect than other wavelet when SNR increases. The types of signal mapping also affect the PAPR performance. The rate of transmission that is the number of bits per symbol depends on the 
modulation schemes scheme used. If the modulation scheme is lower, the data rates is also lower due to less number of bits per symbol. It results in increasing the symbol pulse-width thus decreasing signal bandwidth and a better BER performance. 16 QAM modulation scheme Is used so that the pulse width is fixed here. BER curves for different types of equalizer under different SNR. From the Figure 7, it can be observed that the proposed wavelet has low error rate than the others, thus it produces best transmission quality. The BER performance shows the better results as increases with SNR. After SNR=10, the improvement in BER performance is reduced.
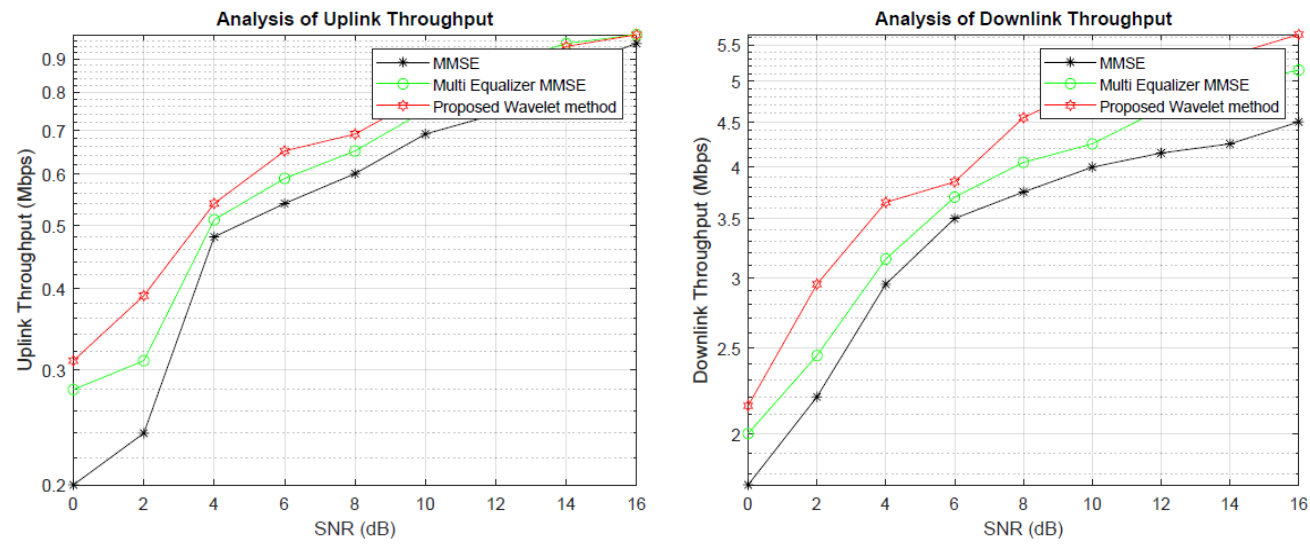

Figure 8 a) Upload throughput b) Download throughput

The maximum data rate of LTE-DWT-OFDM depends on the bandwidth and channel quality condition. Modulation scheme also affects the throughput. The higher modulation scheme transmits more bit per unit time. The wider the bandwidth gives higher the throughput. The upload throughput indicates the maximum data rate of transmission and downlink indicates the maximum data rate of receiver.

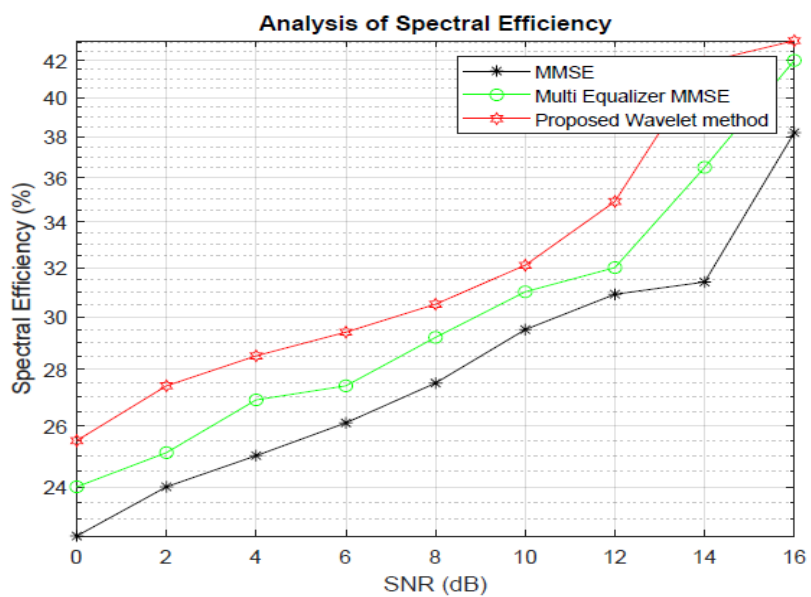

Figure 9. Spectral efficiency vs SNR

Figure 8 shows the upload and downlink performance for $20 \mathrm{MHz}, 16 \mathrm{QAM}$ modulation scheme with different types of wavelet. For FDD, TDD different channel bandwidth is used and channel bandwidth decides maximum data rate. From the Figure 8, it can be perceived that the upload and downlink throughput of the proposed wavelet increases as the SNR increases. The efficiency of the spectrum is calculated using the information rate over the channel bandwidth. The multicarrier modulation with filter bank offers high spectral efficiency. The pulse shaping of QAM is one of the factors that gives profits to spectral efficiency. The spectral frequency of the proposed wavelet increases with SNR when compare to other wavelet as shown in Figure 9.

\section{Conclusion}

The performance of the DWT=OFDM based on Haar wavelet with two equalizer MMSE and MLE is analyzed. The objective of the wavelet is to reduce both Inter Symbol Interference and Inter Carrier Interference through which the performance of the wavelet transform is improved. It can be observed that the performance curve of PAPR, BER, Upload throughput, downlink throughput and spectral efficiency are better than other wavelets. The scaling level of the carrier is taken out as 2 and 16 bit QAM modulation scheme is used for implementation of the modulation technique. In future the performance of the proposed wavelet is studied by increasing the scaling coefficient and by introducing different modulation scheme for modulation technique. 


\section{References}

Sampath H, Talwar S, Tellado J, Erceg V, and Paulraj A. A fourth-generation MIMO-OFDM broadband wireless system: design, performance, and field trial results. IEEE Communications Magazine 2002; 40: 143-149.

García M, Oberli C. Intercarrier Interference in OFDM: A General Model for Transmissions in Mobile Environments with Imperfect Synchronization. Journal of Wireless Communication Network 2009; https://doi.org/10.1155/2009/786040.

Makni, Mariem \& Baklouti, Mouna \& Niar, Smail \& Biglari-Abhari, Morteza \& Abid, Mohamed. Heterogeneous Multi-Core Architecture for a 4G Communication in High-Speed Railway. 2015 10th International Design \& Test Symposium (IDT), Amman, Jordan, 2015, pp. 26-31, doi: 10.1109/IDT.2015.7396731.

Eric Moulines, Pierre Duhamel JF. Cardoso and Sylvie Mayrargue. Subspace methods for the blind identification of multichannel FIR filters Signal Processing. IEEE Transactions on Signal Processing 1995; 43(2):516-525.

Negar Mohaghegh, Shima Kheradmand, and Nahid Ghofrani. An Overview On Equalization Techniques for MIMO-OFDM Systems. International Journal of Wireless Communication and Simulation 2017; 9(1): 1 -14 .

Weinstein SB and Ebert PM. Data transmission by frequency-division multiplexing using the discrete Fourier transform. IEEE Transactions on Communications 1971;19(5):628-634.

Juan- Juan J, Ye- Cai G, Min G and Zhen- Xing L. Orthogonal double wavelet transform based double error function blind optimization algorithm. 2009 International conference on Human machine systems and cybernetics, Hangzhou, China, 2009; pp. 269 - 272. Doi: 10.1109/IHMSC.2009.75.

Massimiliano Martone. Wavelet-based separating kernels for sequence estimation with unknown rapidly timevarying channels. $2^{\text {nd }}$ IEEE Workshop in Signal Processing Advances in Wireless Communications 1995; pp. 255-258, Gaithersburg, MD.

Massimiliano Marton. Wavelet-based separating kernels for array processing of cellular DS/CDMA signals in fast fading Communications. IEEE Transactions on Communications 2000; 48(6):979-995.

Tong Zhou G, Yongsub Kim, and Giannakis GB. Estimation and equalization of time-selective fading channels. Conference Record of the Thirty-Third Asilomar Conference in Signals, Systems, and Computers, 1999; 1:248-252, Atlanta, GA.

Anuradha and Kumar N. BER analysis of conventional and wavelet based OFDM in LTE using different modulation techniques. 2014 Recent advances in Engineering and Computational Sciences, Chandigarh, India, 2014, pp. 1 - 4. Doi: 10.1109/RAECS.2014.6799622.

Kumbasar V and Kucur O. Performance comparison of wavelet based and conventional OFDM systems in multipath Rayleigh fading channels. Digital Signal Processing 2012; 22(5): 841 - 846.

Patra JP, Sing P. Efficient signal detection methods for high mobility OFDM systems with transmit diversity. Arabian Journal of Science and Engineering 2019; 44: 1769 - 1778.

Lin $\mathrm{D}, \mathrm{Wu} \mathrm{X}$ and Sha $\mathrm{X}$. Fractional Fourier Transform based transmitted reference scheme for UWB communications. Science China Information Sciences 2011; 54: 1712. Doi: 10.1007/s11432-011-4209-8.

Nerma MHM, Elmaleeh MAA. PAPR for OFDM system based on fast discrete Curvelet transform. Journal of Engineering Science and Technology 2018; 13(9): 2805 - 2819.

Sandberg SD, Tzannes MA. Overlapped Discrete Multitone Modulation for High Speed Copper Wire Communications. IEEE Journal on Selected Areas in Communications 1995;13(9): 1571-1585.

Achala Deshmukh and Shrikant Bodhe. Comparison of DCT and Wavelet Based OFDM System Working in 60 GHz Band. International Journal of Advancements in Technology 2012; 3(2): 74 - 83.

Al-jawhar, Yasir \& Ramli, Khairun \& Taher, Montadar \& Mohd Shah, Nor Shahida \& Audah, Lukman \& Sami, Mustafa. Zero-padding techniques in OFDM systems", International Journal on Electrical Engineering and Informatics 2019; 10(4): 704-725. Doi: 10.15676/ijeei.2018.10.4.6.

Asif A, Abd-Alhameed RA, Anoh OO, and Dama YAS. Performance Evaluation of DWT-FDM and FFTOFDM for Multicarrier Communications Systems using Time Domain Zero Forcing Equalization. International Journal of Computer Applications 2012; 51(4): 1 - 5.

Li Alex Li, Hua Wei, Yao Yao, Gong Chen, Weiwei Ling, Jiang Du and Yao Huang. Multi-segmental OFDM signals equalization with piecewise linear channel 
model over rapidly time-varying channels. EURASIP Journal on Wireless Communications and Networking 2017; 191. doi: 10.1186/s13638-017-0977-1

Sanyal A and Singh P. BER improvement in OFDM with ZF and MMSE equalizers using ASTC encoder in different fading channels. International Journal of advanced research and science 2014; 1(1): $23-27$.

Sahar Ebadinezhad, Saddam Hasan. BER Evaluation in LTE SC-FDMA under Multipath Channels. International Journal of Recent Technology and Engineering 2019; 8(4): 3539 - 3547.

Narmanlioglu, O, Turan B, Ergen SC, Uysal M. Cooperative MIMO-OFDM based inter-vehicular visible light communication using brake lights. Computer Communications 2018;120: 138-146.

Chafii, Marwa \& Harbi, Yahya \& Burr, Alister. Wavelet-OFDM vs. OFDM: Performance comparison. 23 ${ }^{\text {rd }}$ International conference on Telecommunications, Thessaloniki, Greece, 2016, pp. 1-5. 10.1109/ICT.2016.7500465.

Seyed Mohammad Sajad Sadough, Mahieddine M. Ichir, Pierre Duhamel, and Emmanuel Jaffrot. Waveletbased semi blind channel estimation for ultra-wide band OFDM systems. IEEE Transactions on Vehicular Technology 2009;58(3):1302-1314.

Chen, Pei \& Kobayashi, Hisashi. Maximum likelihood channel estimation and signal detection for OFDM system. 2002 IEEE International conference on communications, New York, NY, USA, 2002; 3: 1640 1645. 10.1109/ICC.2002.997127.

Hamid, Zahraa \& El-Henawy, A. \& Abd El-Samie, Fathi \& El-Shenawy, H. \& Samir, Moataz (2012). “On the performance of FFT/DWT/DCT based OFDM systems with Chaotic Interleaving and Channel Estimation Algorithms. Wireless Personal Communications 2014;78: 1495 - 1510. 